\title{
REGULARITY AND POSITIONAL GAMES
}

BY

\section{A. W. HALES AND R. I. JEWETT}

1. Introduction. Suppose $X$ is a set, $\mathscr{S}$ a collection of sets (usually subsets of $X$ ), and $N$ is a cardinal number. Following the terminology of Rado [1], we say $\mathscr{S}$ is $N$-regular in $X$ if, for any partition of $X$ into $N$ parts, some part has as a subset a member of $\mathscr{S}$. If $\mathscr{S}$ is $n$-regular in $X$ for each integer $n$, we say $\mathscr{S}$ is regular in $X$.

For example, let $X=\{1,2, \cdots, m n-n+1\}$ and $\mathscr{S}$ be all $m$ element subsets of $X$ (hereafter designated $X^{(m)}$ ). Then $\mathscr{S}$ is $n$-regular in $X$, but not $(n+1)$ regular. Another example is the famous theorem of Ramsey which states that given integers $k, m, n$, there exists an integer $p$ such that, if $A=\{1,2, \cdots, p\}$, then $\left\{B^{(k)}: B \in A^{(m)}\right\}$ is $n$-regular in $A^{(k)}$. The concept of regularity is useful in analyzing certain types of games, as we shall see in $\S 3$. In $\S 2$, we shall give some general results and discuss related problems.

2. Regularity. One of the first problems in this area was proposed at Göttingen in 1927. The problem was as follows: If the positive integers are split into two parts, does one part contain arithmetic progressions of arbitrary length? B. L. van der Waerden solved this and a more general problem. He proved that, given integers $m$ and $n$, there exists an integer $p$ such that the set of all arithmetic progressions of length $m$ is $n$-regular in $\{1,2, \cdots, p\}$ [2]. This will be a consequence of Theorem 1. First we shall give some preliminaries.

Definition. If $\mathscr{S}$ and $\mathscr{T}$ are collections of sets, let $\mathscr{S} \otimes \mathscr{T}$ be the collection of all sets $A \times B$, where $A$ is in $\mathscr{S}$ and $B$ is in $\mathscr{T}$.

LEMMA 1. Let $M$ and $N$ be cardinal numbers. Let $\mathscr{S}$ be $N$-regular in $X$, a set of cardinality $M$, and let $\mathscr{T}$ be $N^{M}$-regular in $Y$. Then $\mathscr{S} \otimes \mathscr{T}$ is $N$-regular in $X \times Y$.

Proof. Let $P$ be a set of cardinality $N$. Then a partition of $X \times Y$ into $N$ parts can be represented by a function $f$ from $X \times Y$ into $P$. For each $y \in Y$, $f$ defines a function $f_{y}$ from $X$ into $P$ given by

$$
f_{y}(x)=f(x, y) .
$$

Since there are $N^{M}$ such functions the mapping $y \rightarrow f_{y}$ induces a partition of $Y$ into $N^{M}$ parts. One of these parts contains as a subset a member $T$ of $\mathscr{T}$. That is, for all $y, y^{\prime} \in T$

Received by the editors July 5, 1961 and, in revised form, December 26, 1961. 


$$
\begin{aligned}
f_{y} & =f_{y^{\prime}}, \\
f(x, y) & =f\left(x, y^{\prime}\right) \quad(x \in X) .
\end{aligned}
$$

Choose $y_{0} \in T$. Then $f_{y_{0}}$ partitions $X$ into $N$ parts and hence $\exists S \in \mathscr{S}, p \in P$ such that

$$
f_{y_{0}}(x)=p
$$

But then

$$
f_{y}(x)=p \quad(x \in S, y \in T) .
$$

That is,

$$
f(x, y)=p \quad(x \in S, y \in T)
$$

which was to be shown.

Definition. Let $X$ and $Y$ be sets, $\mathscr{S}$ and $\mathscr{T}$ collections of sets. Then a mapping $f: X \rightarrow Y$ is called provincial with respect to $\mathscr{S}$ and $\mathscr{T}$ in case when $A \in \mathscr{S}$, $A \subseteq X$ there exists a set $B \in \mathscr{T}, B \subseteq Y$ such that $B \subseteq f(A)$.

Lemma 2. Let $f: X \rightarrow Y$ be provincial with respect to $\mathscr{S}$ and $\mathscr{T}$. Then if $\mathscr{S}$ is $N$-regular in $X, \mathscr{T}$ is $N$-regular in $Y$.

Proof. Let $P$ be a set of cardinality $N$ and $g: Y \rightarrow P$. Then $g(f): X \rightarrow P$ and there exist $p \in P$ and $A \in \mathscr{S}$ such that $A \subseteq X$ and $g(f(A))=\{p\}$. But there is a $B \in \mathscr{T}, B \subseteq Y$ such that $B \subseteq f(A)$. So $g(B)=\{p\}$.

LEMMA 3. Let $X$ be a semigroup, $\mathscr{S} \subseteq 2^{X}$. Suppose for each positive integer $k, \mathscr{S}$ is $k$-regular in a finite subset of $X$. Then for each $n$,

$$
\mathscr{S}_{n}=\left\{A_{1} A_{2} \cdots A_{n}: A_{i} \in \mathscr{S}\right\}
$$

is regular in $X$.

Proof. We induct on $n$. Suppose $\mathscr{S}_{n-1}$ is regular in $X$ and $k \geqq 1$. Then there is an integer $m$ and $B \in X^{(m)}$ such that $\mathscr{S}$ is $k$-regular in $B$. Since $\mathscr{S}_{n-1}$ is $k^{m}$ regular in $X, \mathscr{S} \otimes \mathscr{S}_{n-1}$ is $k$-regular in $B \times X \subseteq X \times X$. But the mapping $(x, y) \rightarrow x y$ of $X \times X$ into $X$ is clearly provincial with respect to $\mathscr{S} \otimes \mathscr{S}_{n-1}$ and $\mathscr{S}_{n}$, and thus $\mathscr{S}_{n}$ is $k$-regular in $X$.

Let $W$ be a fixed set and $t \notin W$. Let $X$ be the free semigroup on the set $W$. A functional $f$ is a mapping of $W$ into $X$ which can be described as follows. For some positive integer $n$ there is an $n$-tuple $\alpha=\left(\alpha_{1}, \alpha_{2}, \cdots, \alpha_{n}\right)$ of elements of $W \cup\{t\}$ in which $t$ appears at least once, such that, for $w \in W, f(w)$ is the result of replacing the $t$ by a $w$ and multiplying (in $X$ ) the $n$ components of the new $n$-tuple.

For example, if $W=\{1,2,3,4\}$ and $\alpha=(1, t, 3, t, t, 2,1, t)$ then the corresponding functional $f$ would satisfy

$$
f(4)=14344214
$$


Suppose that $f_{1}, f_{2}, \cdots, f_{n}$ are functionals. Let $\phi: W^{n} \rightarrow f_{1}(W) f_{2}(W) \cdots f_{n}(W)$ be defined by

$$
\phi\left(w_{1} w_{2} \cdots w_{n}\right)=f_{1}\left(w_{1}\right) f_{2}\left(w_{2}\right) \cdots f_{n}\left(w_{n}\right) .
$$

We see that if $g$ is a functional of "length" $n$ then there is a functional $h$ such that

$$
\phi(g(w))=h(w)
$$

Loosely, we have $\phi(g(t))=h(t)$.

If $A \subseteq W, R \subseteq W^{n}$ and $R$ is a member of $\{f(A): f$ is a functional $\}$ then $\phi(R)$ is also a member of this collection. Thus, relative to this collection, $\phi$ is a provincial map of $A^{n}$ onto $f_{1}(A) f_{2}(A) \cdots f_{n}(A)$.

THEOREM 1. If $A$ is a finite subset of $W$ the collection $\{f(A): f$ is a functional\} is regular in $X$.

Proof. Let $\left(I_{m, j}\right)$ be the statement: If $B \in W^{(m)}$ there exists an integer $p$ such that $\{f(B): f$ is a functional $\}$ is $j$-regular in $B^{p}$.

We will prove these statements by induction and thus prove the theorem. It is clear that $\left(I_{m, 1}\right)$ and $\left(I_{1, j}\right)$ are true. Assume further that for $n>1$ and $k \geqq 1$, $\left(I_{n, k}\right)$ and $\left(I_{n-1, j}\right)$ are true for all $j$.

Let $A \in W^{(n)}$. Pick $a \in A$ and let $B=A-\{a\}$. By assumption, there is an integer $r$ such that $\{f(A): f$ is a functional $\}$ is $k$-regular in $A^{r}$. By $\left(I_{n-1, j}\right)$, the fact that $B^{s}$ is finite for each $s$, and Lemma 3 , we see that $\left\{f_{0}(B) f_{1}(B) \cdots f_{r}(B): f_{i}\right.$ is a functional $\}$ is $(k+1)$-regular in the subsemigroup of $X$ generated by $B$, namely, $B^{1} \cup B^{2} \cup \ldots$ $\cup B^{s} \cup \cdots$. The $B^{s}$ are disjoint, and if $f_{0}, f_{1}, \cdots, f_{r}$ are functionals, then the set $f_{0}(B) f_{1}(B) \cdots f_{r}(B)$, all of whose elements have the same "length," meets at most one of the $B^{s}$. In such a situation, $(k+1)$-regularity in the union implies $(k+1)$ regularity in one of the parts. Thus there is an integer $q$ such that

$$
\left\{f_{0}(B) f_{1}(B) \cdots f_{r}(B)\right\}
$$

is $(k+1)$-regular in $B^{q}$. We will use the integer $q$ to verify $\left(I_{n, k+1}\right)$.

Let $A^{q}=P_{0} \cup P_{1} \cup \ldots \cup P_{k}$. This defines a partition of $B^{q}$ and so there are functionals $g_{0}, g_{1}, \cdots, g_{r}$ such that $g_{0}(B) g_{1}(B) \cdots g_{r}(B)$ is contained in one of the parts, say $P_{0}$, and also in $B^{q}$. Thus each "entry" in a $g_{i}$ is an element of $B \cup\{t\}$. Since $B \subseteq A$, we can conclude that $g_{0}(A) g_{1}(A) \cdots g_{r}(A) \subseteq A^{q}$. Now the mapping $\phi: A^{r} \rightarrow g_{0}(a) g_{1}(A) \cdots g_{r}(A)$ defined by $\phi\left(w_{1} w_{2} \cdots w_{r}\right)=g_{0}(a) g_{1}\left(w_{1}\right) \cdots g_{r}\left(w_{r}\right)$ is provincial with respect to $\{f(A): f$ is a functional $\}$. Thus, by $\left(I_{n, k}\right),\{f(A)\}$ is $k$ regular in $g_{0}(a) g_{1}(A) \cdots g_{r}(A)$.

If $g_{0}(a) g_{1}(A) \cdots g_{r}(A)$ is disjoint from $P_{0}$ we are done. If not, there are elements $a_{1}, a_{2}, \cdots, a_{r}$ of $A$ such that

$$
x=g_{0}(a) g_{1}\left(a_{1}\right) \cdots g_{r}\left(a_{r}\right) \in P_{0} .
$$

Suppose $x=v_{1} v_{2} \cdots v_{q} \in A^{q}$. Define $\alpha=\left(\alpha_{1}, \alpha_{2}, \cdots, \alpha_{q}\right)$ by 


$$
\alpha_{i}= \begin{cases}v_{i} & \text { if } v_{i} \in B \\ t & \text { if } v_{t}=a\end{cases}
$$

Note that $t$ appears in $f_{0}$, so $a$ appears in $f_{0}(a)$, and hence $t$ appears in $\alpha$. Then $\alpha$ represents a functional $g$ for which

$$
g(a)=x
$$

and $g(B) \subseteq g_{0}(B) g_{1}(B) \cdots g_{r}(B) \subseteq P_{0}$. Since $g(A)=g(B) \cup\{g(a)\}$, we have $g(A) \subseteq P_{0}$, and the theorem is proved.

COROllary. Let $S$ be a finite subset of a commutative semigroup H. Then the collection of all sets

$$
\{a+n x: x \in S\}
$$

where $a \in H$ and $n$ is a positive integer, is regular in $H$.

Proof. Let $X$ be the free semigroup on $S$. Then the mapping

is provincial.

$$
a_{1} a_{2} \cdots a_{n} \rightarrow a_{1}+a_{2}+\cdots+a_{n}
$$

COROLlARY (VAN DER WAERDEN). For any partition of the positive integers into a finite number of parts, one of the parts contains arithmetic progressions of arbitrary length.

The stronger statement proved by van der Waerden and mentioned above is clear from the proof of Theorem 1. This suggests a general result which we have given as a corollary to Theorem 2. Theorem 2 is proved in Rado [3]. The proof is essentially an application of Tychonoff's Theorem, as shown by Gottschalk [4].

THEOREM 2. Let $X$ and $\Gamma$ be sets, and for every finite subset $A$ of $X$ let $f_{A}$ be a function from $A$ into $\Gamma$. Suppose that for each $x \in X, \Gamma_{x}=\left\{f_{A}(x): A\right.$ is a finite subset of $X$ containing $x\}$ is finite. Then there exists a function $F$ from $X$ into $\Gamma$ with the property that, given any finite subset $A$ of $X$, there exists a finite subset $B$ of $X$ such that $F$ and $f_{B}$ agree on $A$.

Corollary. Let $X$ be a set and $\mathscr{S}$ a collection of finite sets. Then if $\mathscr{S}$ is $n$ regular in $X$ for some positive integer $n, \mathscr{S}$ is $n$-regular in a finite subset of $X$.

Proof. Let $P$ be a set with $n$ elements. Suppose $\mathscr{S}$ is not $n$-regular on any finite subset of $X$. Then for each finite subset $A$ of $X$, there is a function $f_{A}$ from $A$ into $P$ that is not constant on any member of $\mathscr{S}$. By Theorem 2 there is a function $F$ from $X$ into $P$ that is not constant on any member of $\mathscr{S}$, a contradiction.

The above corollary suggests a general problem. Let $M$ and $N$ be cardinals. Does there exist a cardinal $P$ having the following property? If $X$ is a set and $\mathscr{S}$ is a collection of sets each of cardinality less than $M$, and $\mathscr{S}$ is $N$-regular in 
$X$, then $\mathscr{S}$ is $N$-regular in a subset of $X$ of cardinality less than $P$. A simple example shows that if $M>1$ and $N>1$ are integers, $P=\aleph_{0}$ is best possible. The corollary says that if $M=\aleph_{0}$, and $N>1$ is finite, then $P=\aleph_{0}$ is sufficient. No further results in this area are known.

Another problem is the "rectangle" problem. Let $M, N$, and $P$ be cardinals. For what pairs $(R, S)$ of cardinals (if any) is the following true? If $X$ has cardinality $R$ and $Y$ has cardinality $S$, then $X^{(M)} \otimes Y^{(n)}$ is $P$-regular in $X \times Y$. That is, if an $R \times S$ rectangle is partitioned into $P$ parts, one part contains an $M \times N$ rectangle. From Lemma 1, such pairs always exist. For example, if $M=2, P=2$, $N=5, P=2$, then $R=3$ and $S=40$ is sufficient. The "minimal" pairs $(R, S)$ for given $M, N$, and $P$ are not known in general. A particularly interesting case occurs when $M=N=\aleph_{0}$ and $P=2$. It is easily seen that $\left(\aleph_{0}, \aleph_{0}\right)$ does not work and $\left(\aleph_{0}, 2^{2^{\aleph_{0}}}\right)$ does. The sufficiency of $\left(\aleph_{0}, 2^{\aleph_{0}}\right)$ or, for that matter, $\left(2^{\aleph_{0}}, 2^{\aleph_{0}}\right)$ is an open question.

3. Positional games. By a positional game we shall mean a game played by $n$ players on a "board" (finite set) $X$ with which is associated a collection $\mathscr{S}$ of subsets of $X$. The rules are that each player, in turn, claims as his own a previously unclaimed "square" (element) of $X$. The game proceeds either until one player has claimed every element of some $S \in \mathscr{S}$, in which case he wins, or until every element has been claimed, but no one has yet won, in which case the game is a tie. The most familiar example of such a game is "Tick-TackToe." Another is the Oriental game "Go Moku."

It is known from game theory that, in a finite two-player perfect information game, either one player has a forced win or each player can force a tie [5].

LEMMA 4. In a positional game involving 2 players, where $\mathscr{S}$ is 2-regular in $X$, the first player has a forced win.

Proof. Since no tie can occur, one player has a forced win. Assume the second player has a forced win. But then the first player can force a win by (1) making his first move at random, and (2) thereafter following the optimum strategy for the second player, ignoring the last random move, and playing again at random if this is impossible. Since having made an extra move cannot possibly hurt, this will give the first player a win, a contradiction. Therefore, the first player has a forced win.

The following result in combinatorial analysis is due to Philip Hall [6].

LEMMA 5. Let $S_{1}, S_{2}, \cdots, S_{n}$ be an indexed collection of finite sets. Then (A) and (B) are equivalent.

(A) There exist $s_{1}, s_{2}, \cdots, s_{n}$ such that each $s \in S_{i}$ and $s_{i} \neq s_{j}$ if $i \neq j$.

(B) For each $F \subseteq\{1, \cdots, n\}$, the set $\bigcup_{i \in F} S_{i}$ has at least as many elements as $F$. 
If condition (A) is satisfied, we say $S_{1}, \cdots, S_{n}$ have distinct representatives. We will use this lemma to exhibit a tying strategy for the second player in certain positional games.

LEMMA 6. Let $X$ be the board of a 2-player positional game with winning sets $\mathscr{S}=\left\{S_{1}, \cdots, S_{n}\right\}$. For $k=1,2, \cdots, n$ let $T_{2 k-1}=T_{2 k}=S_{k}$. Then if $T_{1}, \cdots, T_{2 n}$ have distinct representatives, the the second player can force a tie.

Proof. Let the representatives be $t_{1}, t_{2}, \cdots, t_{2 n}$. Consider the sets $\left\{t_{1}, t_{2}\right\}$, $\left\{t_{3}, t_{4}\right\}, \cdots,\left\{t_{2 n-1}, t_{2 n}\right\}$. Observe that in order to win the first player must have both elements of at least one of these sets. Since the second player can easily prevent this, he can force a tie.

LEMMA 7. Let $\mathscr{S} \subseteq 2^{X}$, where $X$ is finite. Let $n$ be the size of the smallest member of $\mathscr{S}$. Let $m$ be the size of the largest set of the form $\{S \in \mathscr{S}: x \in S\}$ where $x \in X$. If $n \geqq 2 m$, then in the corresponding 2-player positional game, the second player can force a tie.

Proof. By a simple counting argument, Lemmas 5 and 6 can be applied to obtain the desired result.

The rest of this paper will be concerned with a particular class of 2-player positional games, namely generalizations of Tick-Tack-Toe. The traditional Tick-Tack-Toe game is played on a $3 \times 3$ array of points in the plane. For positive integers $k$ and $n$, the " $k$-game" is played on a $k \times k \times \cdots \times k$ ( $n$ times) array of points in $n$-space. If we choose as a board the set

$$
X=\left\{\left(a_{1}, a_{2}, \cdots, a_{n}\right): 1 \leqq a_{i} \leqq k \text { for all } i\right\},
$$

then $S$ is in $\mathscr{S}$, the collection of winning sets (paths), in case $\mathscr{S}$ consists of $k$ points in a straight line. An equivalent characterization of $S \in \mathscr{S}$ would be that the elements of $S$, in some order, are $\alpha_{1}, \alpha_{2}, \cdots, \alpha_{k}$ where $\alpha_{i}=\left(a_{i 1}, \cdots, a_{i n}\right)$ and, for each $j$, the sequence $\left(a_{1 j}, a_{2 j}, \cdots, a_{k j}\right)$ is one of the following:

$$
\begin{aligned}
& (1, \quad 1, \cdots, 1) \\
& (2, \quad 2, \cdots, 2) \\
& \begin{array}{lll}
\cdot & \cdot & \cdot \\
\cdot & \cdot & \cdot \\
\cdot & \cdot & \cdot
\end{array} \\
& (k, \quad k, \cdots, k) \\
& (1, \quad 2, \cdots, k) \\
& (k, k-1, \cdots, 1) \text {. }
\end{aligned}
$$

In this case we say $\alpha_{1}, \alpha_{2}, \cdots, \alpha_{k}$ are in a natural order (there are two such orders).

In traditional Tick-Tack-Toe, the second player can achieve a tie. In the $3^{3}$-game, however, the first player has a forced win (in fact, no tie position exists). 
Thus, in the 3-dimensional games sold on the market, $k$ is usually 4 . Our previous results enable us to draw some conclusions about the existence of winning and tying strategies in the general case.

THEOREM 4. (a) If $k \geqq 3^{n}-1$ ( $k$ odd) or if $k \geqq 2^{n+1}-2$ ( $k$ even), then the second player can force a tie in the $k^{n}$-game.

(b) For each $k$, there exists $n_{k}$ such that the first player can force a win in the $k^{n}$-game if $n \geqq n_{k}$.

Proof. (a) If $k$ is odd, there are at most $\left(3^{n}-1\right) / 2$ paths through any point and this bound is achieved only at the center point. If $k$ is even, the bound is $2^{n}-1$. The result follows readily from Lemma 7 . This suggests that the center point is the optimum move for the first player if $k$ is odd.

(b) In Theorem 1 , let $W=\{1,2, \cdots, k\}$. Note that if $f$ is a functional then $f(W)$ is a path, but the converse is not true. Now $\left(I_{k, 2}\right)$ and Lemma 4 yield the result.

We conjecture that the bounds in Theorem 4(a) can be improved by a direct application of Lemmas 5 and 6 . It seems possible that $k \geqq 2\left(2^{1 / n}-1\right)^{-1}$, i.e., that the total number of points be greater than the total number of paths, can be shown to be sufficient in this way.

Even though, in some $k^{n}$-games, the second player cannot force a tie, a tie position may still exist, i.e., $\mathscr{S}$ (the collection of paths) may not be 2-regular in $X$ (the board). The bounds of Theorem 4(a) apply, but much more can be said.

THEOREM 5. If $k \geqq n+1$, then in the $k^{n}$-game the collection of paths is not 2-regular in the board.

Let $k$ be fixed. For each $n$ let the $k^{n}$-game board $X_{n}$ be the set of $n$-tuples on $\{1,2, \cdots, k\}$. Designate the elements of $G F(2)$ by $\{0,1\}$. Any partition of $X_{n}$ into two parts can be represented (in two ways) as a function from $X_{n}$ into $G F(2)$. Let $f: X_{m} \rightarrow G F(2)$ and $g: X_{n} \rightarrow G F(2)$ represent partitions. Then we define $f \oplus g: X_{m+n} \rightarrow G F(2)$ by

$$
(f \oplus g)\left(a_{1}, \cdots, a_{m+n}\right)=f\left(a_{1}, \cdots, a_{m}\right)+g\left(a_{m+1}, \cdots, a_{m+n}\right)
$$

where addition on the right takes place in $G F(2)$. Thus $f \oplus g$ represents a partition of $X_{m+n}$ into two parts. Note that " $\oplus$ " is an associative operation on functions from the $X_{i}$ into $G F(2)$.

Proof of Theorem 5. Let $V_{1}, V_{2}, \cdots, V_{m}$ be $k$-dimensional vectors over $G F(2)$, that is, functions from $X_{1}$ into $G F(2)$. Define

$$
\left(a_{1}, a_{2}, \cdots, a_{k}\right)^{\prime}=\left(a_{k}, a_{k-1}, \cdots, a_{1}\right) \text {. }
$$

Suppose that for each choice of

$$
w_{i} \in\left\{V_{i}, V_{i}^{\prime}\right\}, \quad r_{i} \in G F(2) \quad(i=1, \cdots, k)
$$


the vector

$$
r_{1} w_{1}+r_{2} w_{2}+\cdots+r_{m} w_{m}
$$

is neither all zeros nor all ones. Then from the above discussion it can be seen that

$$
V_{1} \oplus V_{2} \oplus \cdots \oplus V_{m}
$$

represents a partition of $X_{m}$ no part of which contains a path.

The theorem will be proved if for each $k, k-1$ such vectors can be found. The desired constructions are obvious extensions of the following two examples for odd and even $k$.

For $k=5$ :

$$
\begin{aligned}
& (1,0,0,0,1) \\
& (0,1,0,1,0) \\
& (1,0,0,0,0) \\
& (0,1,0,0,0)
\end{aligned}
$$

For $k=6$ :

$$
\begin{aligned}
& (1, \quad 0,0, \quad 0, \quad 0, \quad 1) \\
& (0,1,0,0,1,0) \\
& (1,0,0,0,0,0) \\
& (0,1,0,0,0,0) \\
& (0,0,1,0,0,0) \text {. }
\end{aligned}
$$

\section{REEERENCES}

1. R. Rado, Note on combinatorial analysis, Proc. London Math. Soc. (2) 48 (1943-45), $122-160$.

2. A. Y. Khinchin, Three pearls of number theory, Graylock Press, Rochester, 1952, pp. 11-12

3. R. Rado, Axiomatic treatment of rank in infinite sets, Canad. J. Math. 1 (1949), 338.

4. W. H. Gottschalk, Choice functions and Tychonoff's Theorem, Proc. Amer. Math. Soc. 2 (1951), 172.

5. D. Blackwell and M. A. Girshick, Theory of games and statistical decisions, Wiley, New York, 1954, p. 21.

6. P. Hall, On representatives of subsets, J. London Math. Soc, 10 (1935), 26-30.

California Institute of Technology, Pasadena, California

UNIVERSITY OF OREGON,

EUGENE, OREGON 